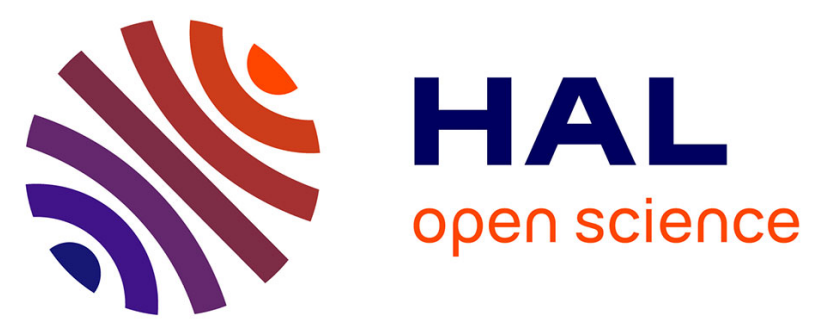

\title{
La Grange-du-Bief à Anse (Rhône) : nouvelles recherches et mosaïques inédites
}

\author{
Henri Lavagne, Richard Tenu
}

\section{To cite this version:}

Henri Lavagne, Richard Tenu. La Grange-du-Bief à Anse (Rhône) : nouvelles recherches et mosaïques inédites. Gallia - Fouilles et monuments archéologiques en France métropolitaine, 1985, 43 (1), pp.147166. 10.3406/galia.1985.2824 . hal-01940141

\section{HAL Id: hal-01940141 \\ https://hal.science/hal-01940141}

Submitted on 27 Feb 2020

HAL is a multi-disciplinary open access archive for the deposit and dissemination of scientific research documents, whether they are published or not. The documents may come from teaching and research institutions in France or abroad, or from public or private research centers.
L'archive ouverte pluridisciplinaire HAL, est destinée au dépôt et à la diffusion de documents scientifiques de niveau recherche, publiés ou non, émanant des établissements d'enseignement et de recherche français ou étrangers, des laboratoires publics ou privés.

\section{(1) (1) $\$$}

Distributed under a Creative Commons Attribution - NonCommercial - NoDerivatives| 4.0 


\title{
LA GRANGE-DU-BIEF A ANSE (Rhône) : NOUVELLES RECHERCHES ET MOSAÏQUES INÉDITES
}

\author{
par Henri LAVAGNE et Richard TENU
}

Il y a vingt-quatre ans, J. Guey et P.-M. Duval ${ }^{1}$ donnaient ici-même une première étude sur la mosaïque la plus importante du site de la Grange-du-Bief, dans la commune d'Anse (Rhône), en attirant l'attention sur la richesse archéologique de cet ensemble encore mal connu, malgré de nombreuses découvertes au cours du Xıx ${ }^{\text {e }} .{ }^{2}$. Par la suite, en 1964-1966, des fouilles fructucuses furent menées au mème emplacement par R. Perraud et $\mathrm{R}$. Auboiron avec l'aide de la Société archéologique du Beaujolais, et publiées dans deux revues malheureusement peu diffusées ${ }^{3}$, mais dont nous reprenons plus loin les informations essentielles. En 1981, à l'occasion d'un projet de lotissement, et sur l'intervention du Directeur des Antiquités historiques de la région Rhône-Alpes, M. J. Lasfargues, des fouilles de sauvetage furent entreprises par une équipe d'archéologues locaux bénévoles, dirigée par R. Tenu. I)epuis $1983^{4}$, après l'acquisition du terrain désormais sauvegardé, le gisement fait l'objet de fouilles programmées et l'analyse archéologique complète en sera conduite à son terme, sans les contraintes de temps qui caractérisaient les autres types d'interventions précédentes ${ }^{5}$. Enfin, des recherches aux Archives des Monuments Historiques à Paris ${ }^{6}$ ont permis de retrouver un dossier daté des années 184j-1847, comprenant une correspondance administrative et scientifique, ainsi que trois dessins inédits concernant les trois pavements mis au jour en 1845 et disparus depuis? Ces documents qui n'avaient

1 J. Guey et P.-.M. Duval., Les mosaïques de la Girange-du-Bief, dans (iallia, 18, 1960, p. 83-102.

2 Celles-ci sont rappelées $i b i d .$, p. 83, n. 1.

3 R. Perrac D, La villa gallo-romaine de la (irange-du-Bief à Anse (Rhône), duns Activiles Beaujolaises, 22, 1965, p. 11-34 el La villa gallo-romaine de la (irange-du-Bief à Anse (Rhône), dans La Physiophile, 68, 1968, p. 3-43. C.f. également de brèves informations dans la chronique de Gallia, 24, 1966, p. 498 et 26, 1968, p. 576-578.

4 L'équipe des fouilleurs tient à remercier M. Lamy, Maire d'Anse, pour sat bienveillante intervention, grâce à laquetle l'açuisition du terrain a pu être réalisée en 1983.

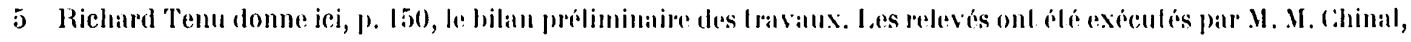
expert-géomètre à Anse.

6 Nous remercions 11 me Bercé, lonservateur en chef des Archives des Monuments Historiques, pour l'eflicacite et la gentillesse avec lesquelles elle a bien voulu guider nos recherches.

7 Sur les avatars de ces mosaïques, infra, p. 156-166. 
1 Anse et ses environs d'apres la carte IGN au 1/250000; : 1, Anse; 2, Lal Grange-du-Bief ; 3, Grange Botton; 4, Grange Baromna ; 5 , Lucenay ; 6, Marcy-sur-Anse; 7 , Lachassagne; 8 , Graves-sur-Anse; 9, Pommiers ; 10, Bourdelan-d'Anse; 11, Amberieux. 
pas été connus des auteurs du Recueil général des mosaïques de la Gaule ${ }^{8}$ lors de la rédaction du fascicule consacré à la civitas des Ségusiaves, ont permis à R. Prudhomme ${ }^{9}$ de restituer le décor de ces mosaïques. Nous avons pensé que l'ensemble de ces éléments nouveaux pouvait donner matière à un second bilan de nos connaissances sur un site déjà considéré comme important par C. Jullian.

Les origines de l'occupation romaine à Anse ne sont pas connues avec précision. Non mentionnée sur la carte de Peutinger, la ville apparaît sur l'Itinéraire d'Antonin sous l'appellation d'Asa Paulini, termes dont l'étymologie a fait l'objet de nombreuses interprétations, peu concluantes en l'absence de documents épigraphiques certains ${ }^{10}$. Son existence doit remonter à la première moitié du ${ }^{\mathrm{e}}{ }^{\mathrm{er}} \mathrm{s}$., si l'on se rapporte à la trouvaille d'un milliaire de Claude daté de $43^{11}$. La création d'une station à Anse est liée à sa situation privilégiée au confluent de la Saône et de l'Azergues (fig. 1). En effet, Anse est une étape fluviale à une trentaine de kilomètres au n. de Lyon, en même temps qu'un carrefour routier sur la voie principale de Lyon à Autun et sur la voie secondaire en direction de Roanne et de la vallée de la Loire. D'autre part, Anse est au centre d'une région renommée pour la fertilité de son sol et de nombreux coteaux ensoleillés ont dû voir la vigne s'implanter très tôt. C'est, enfin, un point stratégique au n. de Lyon, verrou au niveau d'un étranglement naturel entre une boucle de la Saône et les premiers contreforts du Beaujolais. Cies différentes remarques permettent d'affirmer qu'Anse était une place de marché et un "villagecitadelle ", mais aussi très probablement un lieu de villégiature pour quelques notables de la capitale des Trois Gaules. Que reste-t-il aujourd'hui de l'implantation romaine ? Un castellum ou une "forteresse postale", pour reprendre les termes de G. Jullian" ${ }^{12}$, est située au centre de la ville actuelle, où son élévation est encore visible en de nombreux endroits ${ }^{13}$. Le tracé exact de la grande voie de l'Océan et du Rhin qui traversait la cité est très incertain entre Lyon et Anse. Pour la plupart des auteurs ${ }^{14}$, la voie romaine arrivait de Limonest par Les Chères, mais M. C. Guigue ${ }^{15}$ prétend que la route franchissait l'Azergues à la hauteur de Chazay et longeait ensuite la rive gauche de la rivière par Izerable. L'occupation romaine a également laissé dans la région son empreinte dans de nombreux sites qui semblent s'ordonner sur deux couronnes concentriques autour de la ville actuelle. Un premier groupe d'habitats rapprochés (de 800 à 1000 mètres les uns des autres) porte la dénomination de "granges " : Grange Baronna à l'o., Grange Botton au s.-o., Grange-du-Bief au s. Un second

8 H. Stern, Michèle Blanchard-Lemén, Recueil général des mosaïques de la Gaule, Lyonnaise, II, 2, $X^{\mathrm{e}}$ supplémenl à Gallia, 1975, nos 175-179.

9 Ce furent les derniers travaux du dessinateur du Centre de recherches sur la mosaĩque, à la mémoire duquel nous rendons ici hommage. La fig. 11 a été complétée par Patricia Raynaud.

10 J. Guey, P.-M. Duval, op. cit., p. 83, n. 3.

11 Ibid., p. 84, n. 6. J. Descrorx, Sur un nouveau milliaire de Claude, dans Bull. Association lyonnaise de recherches archéologiques, 1937, p. 11 et sq., et P. Wuilleumier, De Lyon à Macon, dans $R E A, 41,1939$, p. 246-247.

12 G. Jullian, Notes gallo-romaines, les problèmes d'Anse-sur-Rhône, dans $R E A$, 26, 1924, p. 69-71.

13 J. Gruyer, L'enceinte romaine d'Anse, études et relevés, Actes du $109^{\mathrm{e}}$ Congrès des Sociétés Savantes, Dijon, 1984, sous presse.

14 A. Audin, Essai sur la topographie de Lagdunum, le confluent et la croisée de Lyon, dans Éludes rhodaniennes, 1947, p. 99 et $R E A, 1951$, p. 97.

15 M. C. Guigue, Les voies antiques du Lyonnais, du Forez, du Beaujolais déterminées par les hôpitaux du Moyen-Age, Lyon, 1877, p. 35. 
ensemble, éloigné de 1500 à $3000 \mathrm{~m}$ du caslellum, correspond à des villages tels qu'Ambérieux au s.-c., Lucenay au s., Marcy-sur-Anse et Lachassagne au s.-o., Graves-sur-Anse à l'o., Pommiers au n.-o., et Bourdelan-d'Anse au n.-c. (fig. 1). Les recherches entreprises ces dernières décennies sur quelques-uns des sites gallo-romains mentionnés ci-dessus ${ }^{16}$ sont malheureusement restées très ponctuelles et sans intégration réelle dans le contexte socio-économique antique de la région.

I. Les fouilles récentes. - L'analyse des sondages récents (fig. 2) a mis en évidence de nombreuses substructions étagées sur la pente de plusieurs terrasses, dont la dénivellation totale est d'environ $18 \mathrm{~m}$.

Terrasse supérieure : les recherches antéricures ont permis d'établir l'existence d'un grand bâtiment de plus de $170 \mathrm{~m}$ de long, reconnu sur $59 \mathrm{~m}$ de large. Une galerie-façade longe l'édifice à l'e. et dessert une série de salles dont les fonctions n'ont pas été clairement définies (salles numérotées de I à IX sur le plan fig. 2). Nos premiers sondages effectués dans la zone s. du bâtiment ont fait apparaître deux parties distinctes, séparées par une zone vierge de tout vestige : au n.-o. nous avons retrouvé (en a sur le plan) le prolongement du mur limite de l'édifice, large de $0,70 \mathrm{~m}$ et arasé à une quinzaine de centimètres au-dessus du niveau du sol d'occupation. Dégagé sur une longueur de $7,80 \mathrm{~m}$, il disparaît brutalement, mais son empreinte en négatif prouve qu'il se poursuivait plus au s. Sa face interne est recouverte d'un enduit blanc de $0,03 \mathrm{~m}$ d'épaisseur et ses fondations de $0,75 \mathrm{~m}$ reposent directement sur une terre argileuse ocre-rouge. Parallèlement à ce mur, une autre substruction détruite jusqu'aux fondations, mais dont la trace apparaît nettement dans l'argile qui l'entoure, limite une zone de $2,90 \mathrm{~m}$ de large où l'on repère un radier de sol à mosaïque. En effet, la couche de démolition, constituée de fragments de mortier et de revêtement mural mêlés à des tuiles brisées, a révélé de nombreuses tesselles de mosaïques noires et blanches. L'absence de retours de murs, d'un quelconque système de chauffage, ou de matériel domestique, laisse supposer l'existence d'un couloir desservant probablement des salles annexes. A l'e. du bâtiment, une zone de $15 \mathrm{~m}$ de large s'appuie contre la galerie-façade (b sur le plan), vaste couloir de 4,40 $\mathrm{m}$ de section. Bien que très arasée, cette zone fait apercevoir deux salles juxtaposées (c et d) dans le prolongement de celles dégagées en 1964 sous la route des Trois Châtels, à quelque $70 \mathrm{~m}$ plus au $\mathrm{n}$. Un second couloir (e sur le plan), de 2,90 m de large, les borde à l'o. Il est limité par un mur de construction identique au mur 0 . du bâtiment et présente une ouverture au $\mathrm{s}$.

Zone médiane : située en contrebas de la terrasse supérieure, elle peut être divisée en quatre grandes zones étagées sur une douzaine de mètres de dénivellation totale.

La première (Zone 1 du plan) jouxte le bâtiment supéricur et est caractériséc, au s., par deux salles VII et VII b, fouillées en 1966-1967, qui présentaient encore à cette époque

16 Notamment celles de h. Perraud, Le sile du Bancillon à Anse, la mosaique Chabert, dans La Physiophile, cit., p. 40-43 et R. Perraud et L. Fraisse, Le sile de Bourdelan d'Anse, dans Résurgences, 1977, 1, p. 2-14. 


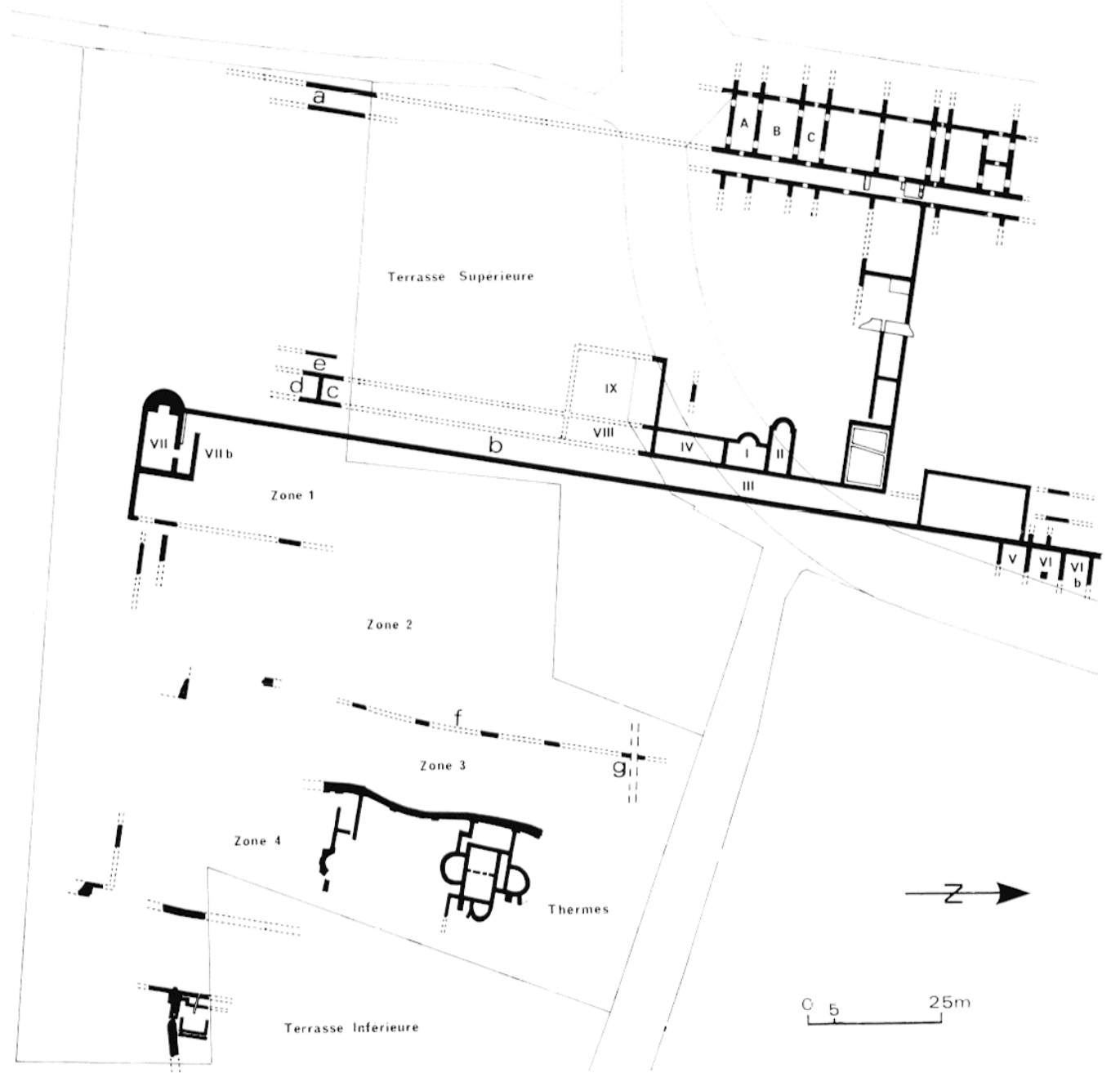

2 Site de la Grange-du-Bief. Plan de siluation où sont reportés les résultats des fouilles antérieures et les découvertes ricentes.

des panneaux de peinture murale fort bien conservés ${ }^{17}$. Nos travaux montrent d'autres substructions à l'e., qui doivent correspondre à une extension des salles précédentes. A environ $20 \mathrm{~m}$ du mur e. de la galeric-façade du bâtiment supéricur, une seconde terrasse (Zone 2) de largeur légèrement plus importante (25 $\mathrm{m}$ ) est bien délimitée à son extrémité orientale par un mur (f) de $0,70 \mathrm{~m}$ d'épaisseur, que nous retrouvons sur une longueur de $60 \mathrm{~m}$. Ces deux zones n'ont fait l'objet que de sondages partiels au n. et au s. par suite de la présence d'un verger et d'une source dans leur partie centrale. La troisième terrasse (Zone 3) s'appuie, à une quinzaine de mètres de la précédente, contre un mur de soutènement de $1,30 \mathrm{~m}$

17 R. Perratd et L. Fraisse, Heurs el malheurs de l'archéologie: les peintures murales de la villa gallo-romaine de la Grange-du-Bief, dans Expansion Beaujolaise, 3, 1972, p. 14-16. 
d'épaisseur en moyenne. Seules quelques substructions sont apparues au s., à proximité de la source, et au n., où l'établissement d'une voie romaine (g) a détruit, en partie, le mur séparant cette zone de sa voisine en amont. La voic romaine, de direction e.-o., est constituée d'un épais blocage et est bordée par un petit caniveau en pierre. La quatrième et dernière zone est nettement mieux définic. Comprise entre deux murs de soutènement distants de $25 \mathrm{~m}$, elle fait actuellement l'objet de fouilles approfondies. Le mur de soutènement o., de belle facture, est orné de contreforts équidistants de 1,40 m de long sur 0,30 m d'épaisseur et présente une courbure prononcée, probablement due à une catastrophe naturelle (fig. 3). Il a été repéré par photographie aérienne sur environ $150 \mathrm{~m}$ vers le $\mathrm{s}$. Le mur de soutènement oriental est très différent puisque constitué de deux constructions de $0,60 \mathrm{~m}$ d'épaisseur accolés l'une à l'autre (fig. 4). Un ensemble thermal tardif, de $800 \mathrm{~m}^{2}$ de superficie (dont la moitié en bâtiments), mis au jour dans la partie n. de la terrasse, fera l'objet d'une prochaine publication, son étude n'étant pas entièrement achevée.

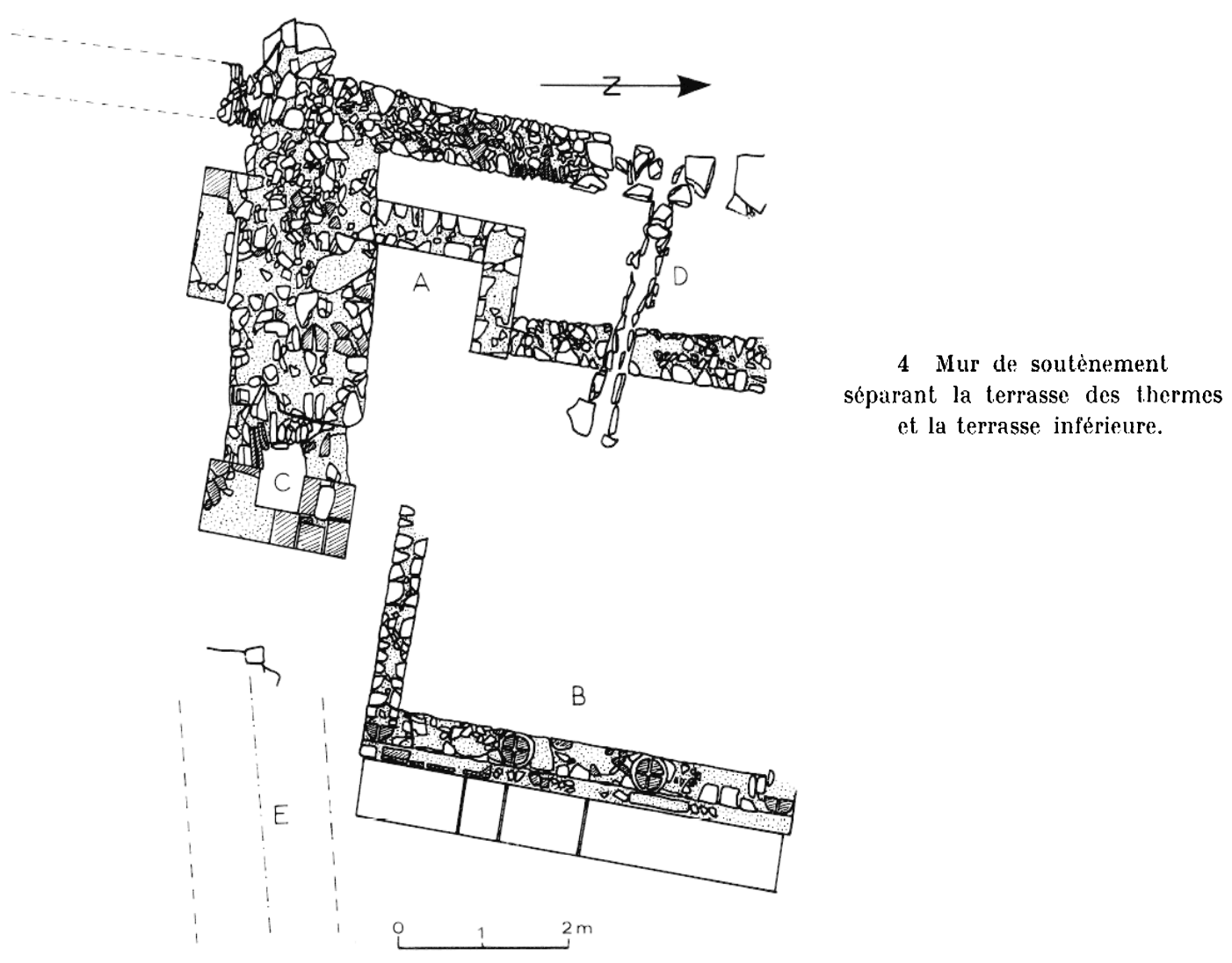

Terrasse inférieure : un sondage d'environ $200 \mathrm{~m}^{2}$, effectué au bas de la pente en bordure des champs cultivés voisins, a dégagé, à $6 \mathrm{~m}$ en contrebas des thermes, un ensemble complexe de substructions imbriquées les unes dans les autres (fig. 5). En l'absence de tout élément de datation précis, seule une chronologie relative a pu être établie. 
La construction la plus ancienne est un petit mur A, de 3,90 m de long, caractérisé par deux retours perpendiculaires de $1,45 \mathrm{~m}$ vers l'e. I'une épaisseur de $0,46 \mathrm{~m}$, son mode de construction est réalisé au moyen d'assises de deux rangées de briques de

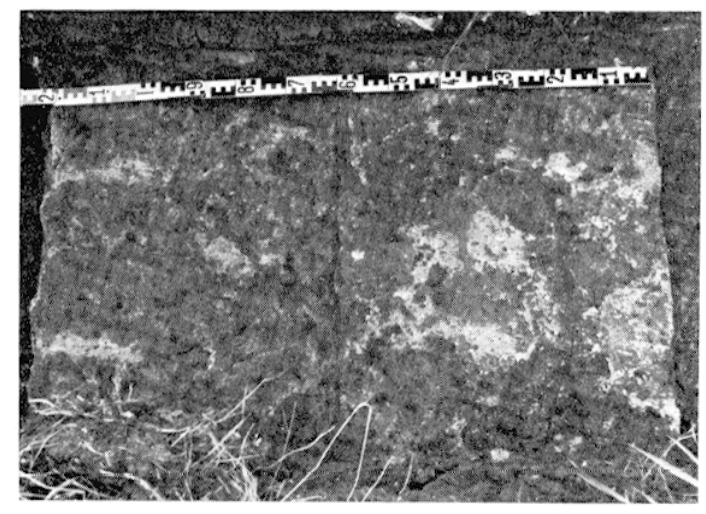

3 Mur de soutenement o. de la terrasse des thermes.

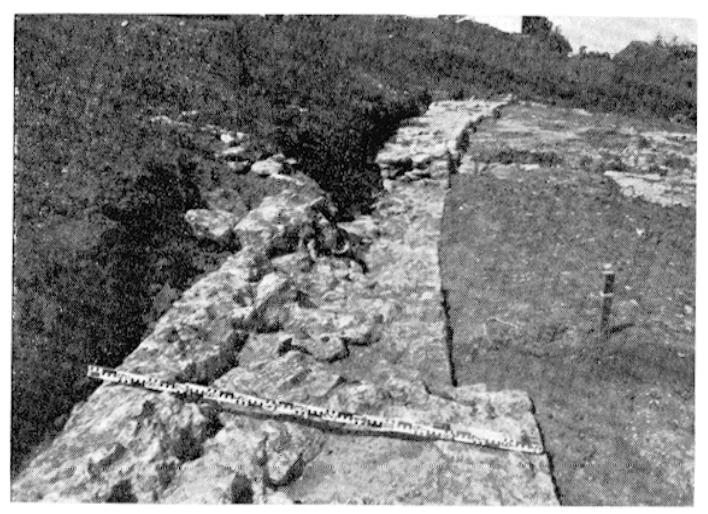

5 Plan des vestiges dégagés dans le sondage de la lerrasse inforienre.

$0,46 \times 0,23 \times 0,08 \mathrm{~m}$ intégrées à des assises de moellons très bien taillés (fig. 6 ). Un conduit voûté $\mathrm{E}$, de direction e.-o., traverse le mur en $\mathrm{U}$ dans son angle s.-o. et constitue extérieurement un épais massif de maçonnerie de $1,80 \mathrm{~m}$ de large. Une rupture de pente et un léger changement de direction ont nécessité la mise en place d'un regard de section carrée $\mathrm{C}(0,53 \times 0,53 \mathrm{~m})$ recouvert sur son pourtour de briques de mêmes dimensions (fig. 7$)$. Dégagé sur 1,10 $\mathrm{m}$ de profondeur, une remontée d'eau n'a pas permis d'en atteindre le fond. Sur chacune des parois internes n. ct s. a été aménagée une cavité au même niveau que

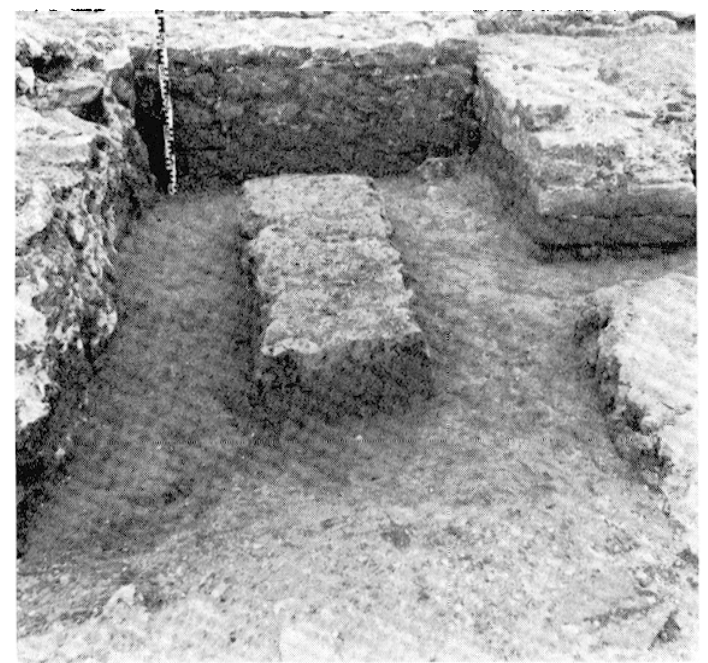

6 Mur avec assises de briques.

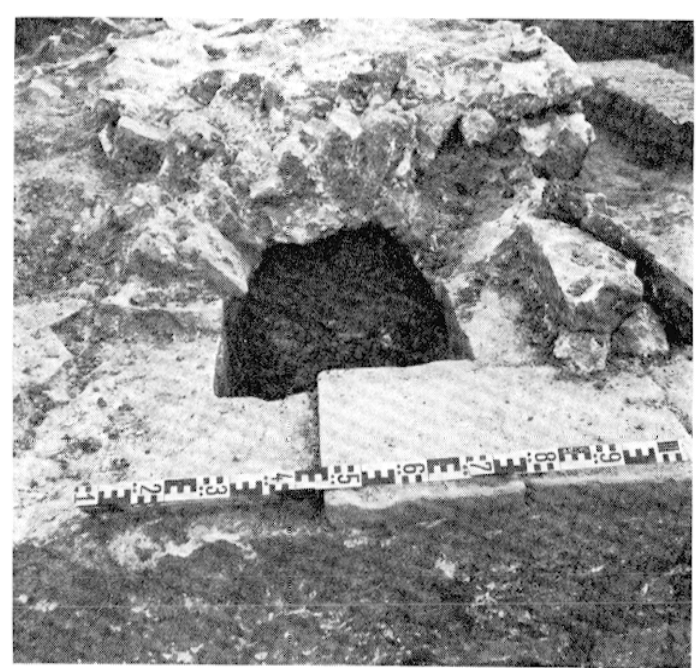

7 Regard dı conduit voûté.

la voûte supérieure du second canal. L'écart entre les intrados des voûtes du canal supérieur et du canal inférieur atteint $0,55 \mathrm{~m}$, la liaison entre cette dernière voûte et le regard se faisant par l'intermédiaire d'un bouchon de pierre demi-circulaire. 
Un second bâtiment $B$ (fig. 8) longe le conduit voûté au n. Il est constitué de deux murs se raccordant à un stylobate de $5,16 \mathrm{~m}$ de long qui supportait initialement quatre colonnes équidistantes : deux colonnes circulaires au centre occupant la largeur totale du stylobate $(0,40 \mathrm{~m})$, les deux autres, semi-circulaires, sont situées aux extrémités. Assemblées au moyen de briques en quart-de-rond (fig. 9), elles étaient recouvertes de stuc peint en "rouge pompéien. " Une rangée de quatre dalles de pierre de $0,64 \mathrm{~m}$ de largeur et de longueur très irrégulière (la plus grande atteint $2,45 \mathrm{~m}$ ) fait face au stylobate à l'e. La partie centrale de ce second bâtiment est couverte d'un remblai de matériaux divers à forte proportion de moellons et d'éclats de picrres, dans lequel se déverse un caniveau en pierre $\mathrm{D}$, lui-même retenu par deux murs parallèles tardifs.

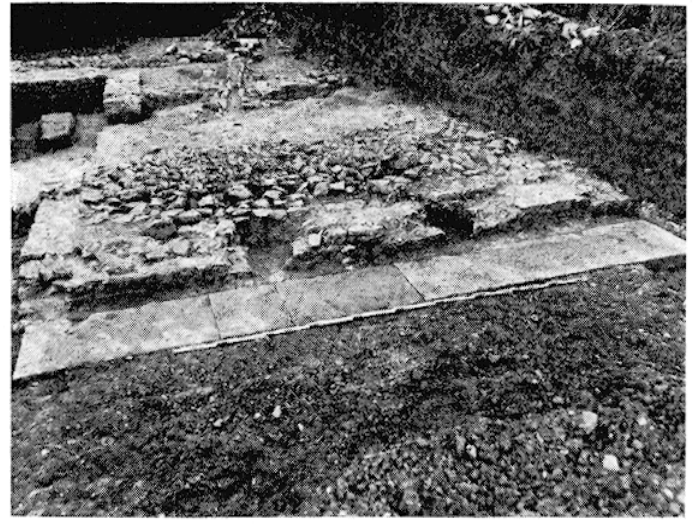

8 Stylobale et rangée de dalles.

9 Colonne circulaire du stylobate. $\rightarrow$

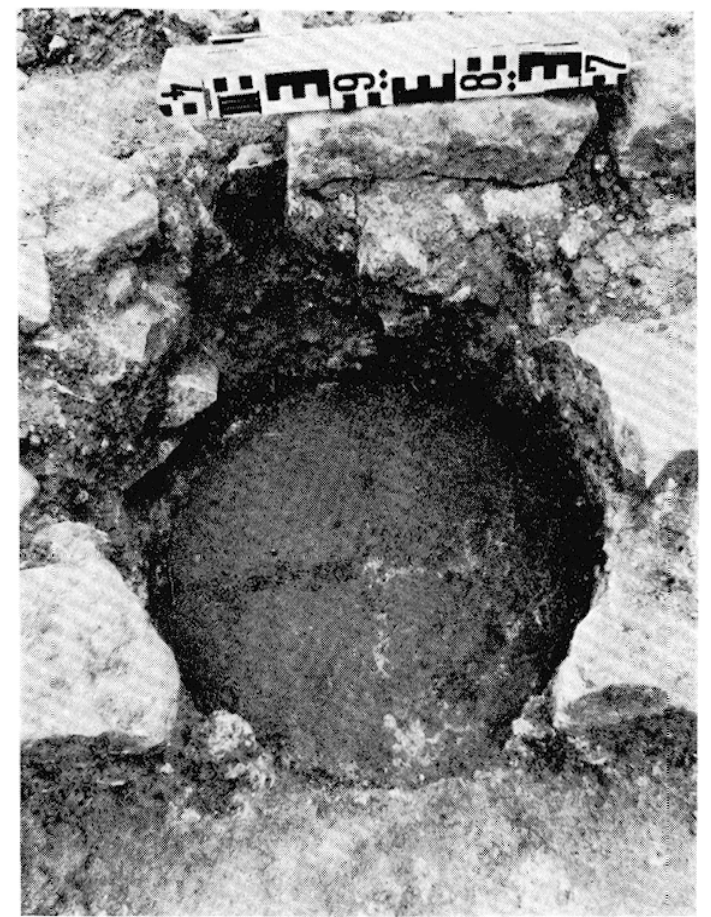

A l'o. de cet ensemble, apparaît une zone très humide qui pourrait correspondre à l'emplacement d'un bassin à en juger par la présence de nombreux fragments de mortier de tuileaux de forte épaisseur.

Aucune stratigraphie n'a été mise en évidence à ce stade des recherches puisque seul le dernier état d'occupation est visible. Notre datation ne peut donc porter que sur la phase d'abandon, bien cernée par la découverte de plusieurs monnaies sur la terrasse supérieure et sur la terrasse des thermes.

Elles indiquent une période post quem au dernier quart du Ive $^{\mathrm{e}} \mathrm{s}$ ou au premier quart du ve s. : un petit bronze à l'effigie de Constantin II (317-337); une médaille commémorative à la Ville de Rome (330-346); un petit bronze à l'effigie de Constance II (337-346); 
un petit bronze à l'effigie de Constance II (346-354); un petit bronze à l'effigie de Valentinien II ? (383-388); deux petits bronzes à l'effigie d'Eugène (392-394); et deux petits bronzes indéchiffrables du $\mathrm{IV}^{\mathrm{e}}$, $\mathrm{v}^{\mathrm{e}} \mathrm{s}$. En outre, deux oboles d'argent de la seconde moitié du XII ${ }^{\mathrm{e}} \mathrm{s}$, dégagées à proximité du mur de soutènement 0 . des thermes, démontrent que le site, encore apparent à cette époque, a dû servir de carrière aux habitants de la ville actuelle.

La céramique, extraite de la couche de terre superficielle, brasséc par les labours ou les plantations, est très fragmentée et très hétérogène, aucune forme complète n'étant reconstituable. Elle corrobore cependant la datation fournie par les monnaies.

Par contre, le type d'architecture est beaucoup plus cohérent. Tous les murs sont constitués d'un blocage interne de pierres et mortier entre deux parements de moellons plus ou moins réguliers, l'épaisseur des joints variant en moyenne entre deux ou trois centimètres. La pierre utilisée est un calcaire oolithique blanc extrait des carrières voisines, celles de Lucenay, célèbres dans toute la basse vallée de la Saône et de l'Azergues.

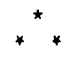

Les sondages effectués dans le terrain appellent plusieurs remarques : la densité de construction est très forte puisque des substructions ont été mises au jour sur la totalité de la superficie du chantier de fouilles ; les structures dégagées tant aujourd'hui que dans les dernières décennies, dénotent une richesse architecturale et ornementale incompatible avec celle d'un habitat rural isolé, où, l'on attendait la construction en murs de terre, courante dans la région; les bâtiments, de dimensions respectables, nous incitent à croire en l'existence d'édifices publics; des éléments du $\mathrm{i}^{\mathrm{er}} \mathrm{s}$. dégagés antérieurement sur la terrasse supérieure, associés à une phase d'abandon à la charnière du Ive et du ve $\mathbf{s}$., attestent une permanence sur environ quatre siècles. Enfin, la vaste étendue du site est confirmée par des photographies aériennes où des vestiges apparaissent sur une quinzaine d'hectares.

Ces constatations font pencher en faveur d'une agglomération importante du type vicus et posent la question fondamentale : sommes-nous à l'emplacement de l'antique Asa Paulini? Nos connaissances sur Anse et la région du Nord Lyonnais sont très fragmentaires, et les problématiques liées à ce type de recherches sont nombreuses. Elles concernent aussi bien le contexte rural que son intégration dans la région et impliquent donc de définir la fonction de l'habitat ct son évolution chronologique, mais également les processus d'urbanisation successifs, l'évolution économique et sociale, le déplacement et le regroupement en un point stratégique. C'est à ces problèmes que devront répondre les nouvelles fouilles actuellement en cours.

Richard Tenu 
II. Les mosaïques de 1845. - Le dossier que nous avons retrouvé aux Archives des Monuments Historiques contient trois dessins inédits, accompagnés d'une correspondance relatant les découvertes et insistant sur la nécessité de prendre des mesures de conservation sur toute l'étendue du site. Lors de la rédaction de leur étude, P.-M. Duval et J. Guey, avaicnt déjà fait mention de certains éléments de ces lettres qui avaient été partiellement publiées dans un article du Bulletin du Comité des Travaux Historiques de l'époque ${ }^{18}$. Comme elles permettent de résoudre le problème de l'emplacement de ces pavements, nous en redonnons ici brièvement la substance.

La première émane du Député-Maire de Lyon, en date du 4 mai 1845, et clle est adressée au Ministre de l'Intérieur (Section des Beaux-Arts). Elle annonce le dégagement de "trois nouvelles mosaïques très voisines de la première ", c'est-à-dire de la mosaïque aux proues de navires (pièce IX) mise au jour deux ans plus tôt. Un croquis succinct donne les dimensions suivantes : première mosaïque (pièce $A$ du plan fig. 2) : 4,60 $\mathrm{m} \times 9 \mathrm{~m}$; deuxième pavement (pièce $\mathrm{B}$ ) : $7 \mathrm{~m} \times 9 \mathrm{~m}$; troisième pavement (pièce $\mathrm{G}$ ) : également $4,60 \mathrm{~m} \times 9 \mathrm{~m}$. Ce sont donc trois pièces contiguës, séparées par des «murs de refend ». On comprend l'attention que le Maire de Lyon porte à ces mosaïques, car à cette époque, le Musée de Lyon, à l'instigation de François Artaud ${ }^{19}$, son fondateur, avait déjà réuni les plus beaux pavements de la Gaulc du Centre, et il est probable que l'idée de leur dépose ne lui est pas étrangère. Une scconde lettre, plus longue, est écritc le 29 mai de la même année au Ministre, par A. Comarmond, Conservateur du Musće archéologique de Lyon et Inspecteur des Monuments Historiques du Rhône et de l'Ardèche. On sait également l'intérêt que ce dernier portait à la mosaïque et $\mathrm{Ph}$. Fabia ${ }^{20}$ a montré combien il a travaillé à enrichir le Musée de Lyon en faisant pratiquer le transfert de pavements lyonnais et viennois. La lettre de Comarmond reprend les termes d'une lettre, à lui adresséc, par J. F. Peyré (26 mai 1845) ${ }^{21}$, conseiller général du Rhône, qui avait déjà acquis la première mosaïque de 1843 et l'avait fait protéger par un abri in situ $u^{22}$. Il ressort que ces trois pavements contigus sont proches d'une quatrième mosaïque "que le travail de la charrue a détruite et dispersée " immédiatement ${ }^{23}$. Peyré, convaincu que les trois mosaïques ne forment qu'un scul "ensemble tripartite», unique par ses dimensions globales, estime "qu'il faut sauver ce monument qui offre plus d'intérêt encore que celui de 1843 et qui est d'ailleurs dans un très bon état de conservation ». Il mentionne de précieux détails de l'iconographie : "oiseaux, dauphins, vases aux formes élégantes, où brillent les marbres noir, blanc, rouge, bleu, jaune et vert ». Mais le document le plus important est le rapport en quatre pages de deux professeurs d'architecture à l'École des Beaux-Arts de Lyon, D. Chenavard et E. Rey, en date du 20 Juillet 1845. Celui-ci comporte des mesures et des

18 J. Guey, P.-M. Duvar, op. cit., p. 85 (cf. BACTII, 1844-1845, p. 486).

19 Sur ce personnage, le plus ancien spécialiste des mosaïques gallo-romaines, cf. nolre article François Arlaud, un précurseur, dans Archeologia, n 15, mars-avril 1976, p. 112-113.

20 Ph. FABIA, Les mosaíques romaines du Musée de Lyon, Iyon, 1923, p. 172.

21 Reprise dans $B A C T H, 1844-1845$, p. 486.

22 Le båtiment est encore en place aujourd'hui et mériterait d'être conservé. C'est un rare témoignage pour ıne étude de muséographie sur la protection des monuments anliques au $\mathrm{XIX}^{\mathrm{e}} \mathrm{s}$., qui reste encore à écrire.

$23 \quad$ No 179 du Recueil général des mosaïques de la Gaule, II, 2. 
observations qui n'avaient pas encore été fournies. On note d'abord que les trois pavements sont situés «à 43 mètres de distance à l'occident de la première " (la mosaïque de la pièce IX), précision qui nous permet de les localiser. Les dimensions des deux salles A et $\mathrm{C}$ sont légèrement différentes $(9,50 \mathrm{~m} \times 4,50 \mathrm{~m})$ de celles que donnait la lettre du Maire de Lyon mais ces variantes sont mineures car, au XIX ${ }^{\mathrm{e}} \mathrm{s}$., on constate que les relevés tiennent compte, ou omettent fréquemment le "raccord " en mosaïque qui lie le tapis aux murs d'une pièce. Les trois dessins aquarellés qu'ont exécutés (henavard et Rey sont entoilés. Le premier $(70 \mathrm{~cm} \times 35 \mathrm{~cm})$ est le plan général des structures qu'ils ont fait dégager autour des salles $\mathrm{A}$, $\mathrm{B}$ et $\mathrm{C}^{24}$. On note au $\mathrm{n}$. la présence de 5 nouvelles pièces, et d'un couloir, apparemment sans mosaïques, séparées de six autres salles par un corridor de service, mais dont les fouilleurs ne paraissent avoir vu que les amorces. Remarquons que ce couloir est placé dans le prolongement exact du tronçon dégagé par R. 'Tenu en 1981 (au point a du plan fig. 2), ce qui donne une confirmation sur la fidélité du relevé. Le second dessin (fig. 10) est un détail "à grandeur d'exécution " $(102 \mathrm{~cm} \times 115 \mathrm{~cm})$ de la mosaïque centrale $\mathrm{B}:$ on y voit un hexagone chargé du motif classique du dauphin accosté d'un trident. Le troisième dessin $(98 \mathrm{~cm} \times 95 \mathrm{~cm})$ est également un détail, en noir et blanc, de la mosaïque A. Avant de revenir sur l'étude des schémas géométriques qu'il est possible de restituer à partir de ces documents, il convient d'élucider le problème de l'emplacement des mosaïques en question.

En effet, R. Perraud ${ }^{25}$ dans ses fouilles de 1967-1968 a cru avoir retrouvé la place de ces trois mosaïques dans les pièces V, VI et VI b du plan (fig. 2). Le fouilleur appuie ses déductions sur trois points : "la largeur totale des trois salles est de $16,20 \mathrm{~m}$ ", ce qui correspond, en effet, à la largeur de ce que l'on appelait «l'ensemble tripartite ». D'autre part, l'une des salles qu'il mit au jour recélait encore des cubes noirs et blancs, comme dans les pièces A ou G mentionnées par la correspondance de 1845. Enfin, le cadastre de 1845 ne porte que deux numéros de terres pour cette zone; or, Peyré déclare que les trois mosaïques furent découvertes dans « une terre voisine de celle sur laquelle reposait la mosaïque de 1843 ». Le plan cadastral ne portant que deux numéros de parcelles (nº 239 et $n^{\circ} 245$ ), on pourrait penser que la mosaïque de 1843 occupait l'une de ces parcelles, l'autre l'étant par les trois pavements de 1845. Enfin, R. Perraud appuie sa conviction sur une remarque de Peyré : des fragments de colonnes en terre-cuite recouvertes de stuc furent dégagés à 3 ou $4 \mathrm{~m}$ au s. des mosaïques, et c'est précisément des éléments analogues que lui-même recueillit dans ses fouilles de 1968. Ces rapprochements sont évidemment troublants et l'on serait tenté de conclure avec le fouilleur que les pièces V, VI et VI b sont bien l'ensemble "tripartite» de 1845. Mais les arguments contraires nous paraissent devoir l'emporter cependant. Les trois mosaïques de 1845 sont "à $43 \mathrm{~m}$ de distance», disent Chenavard et Rey, de la mosaïque aux proues de navires, et précisent-ils, à l'occident Or, les trois salles fouillées par R. Perraud sont situées non pas à $43 \mathrm{~m}$, mais à $73 \mathrm{~m}$, et au n., non pas à l'o., de la première. Peut-on admettre que deux professeurs d'architecture,

24 Malheureusement trop pe'u lisible pour être reproduit ici. Nous en avons reporté les tracés sur le plan de la fig. 2 .

25 Cif. supra, n. 3. 


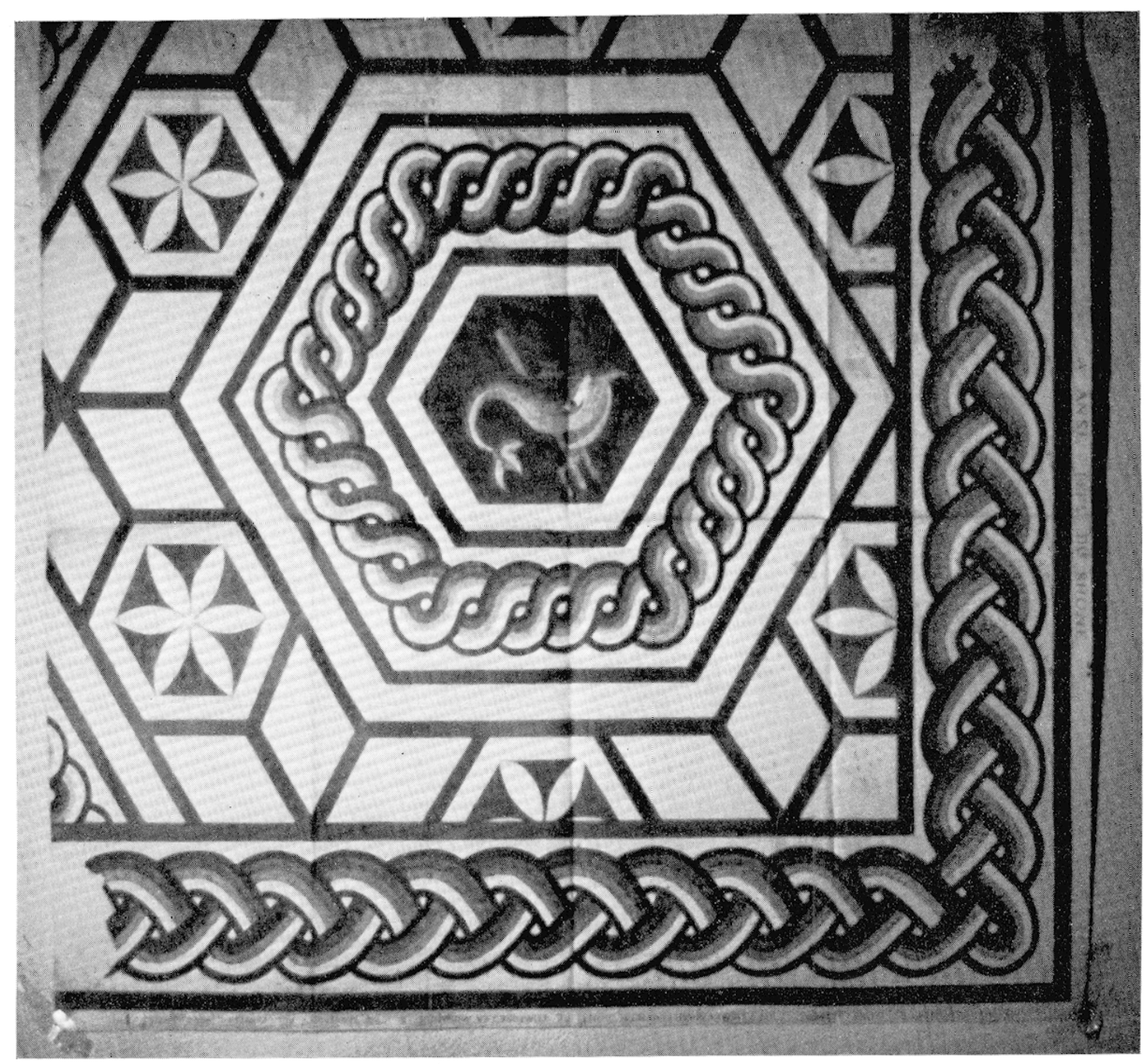

10 Mosaique $n^{\circ}$ 176. Aquarelle Chenavard-Rey (Monuments IIistoriques). Détail d'un angle du pavement.

en principe rompus à l'établissement des relevés, aient confondu le n. et l'o., et porté sur leur plan $43 \mathrm{~m}$ au lieu de 73 ? Nous ne le pensons pas, surtout si l'on rappelle que le couloir qui dessert les pièces $\mathrm{A}, \mathrm{B}, \mathrm{C}$ a été retrouvé dans les sondages ultérieurs. Nous concluons donc, comme H. Stern et Michèle Blanchard-Lemé $c^{26}$, qu'il faut refuser la localisation proposée par R. Perraud, et voir dans les pièces V, VI et VI b, trois nouvelles salles, richement décorées qui n'avaient pas encore été aperçues antéricurement à ses travaux.

Mosäque $n^{0} 176^{27}$ (fig. 10 et 11).

Le commentaire accompagnant le plan levé par Chenavard et liey souligne que "la mosaïque a été exécutée avec un soin remarquable; la petitesse des cubes et leur

26 H. Stern, Michèle Blanchard-I,emée, op. cil., p. 37.

27 Par commodité, nous reprendrons ci-dessous les numéros dı Recueil général des mosaíques de la Gaule. 


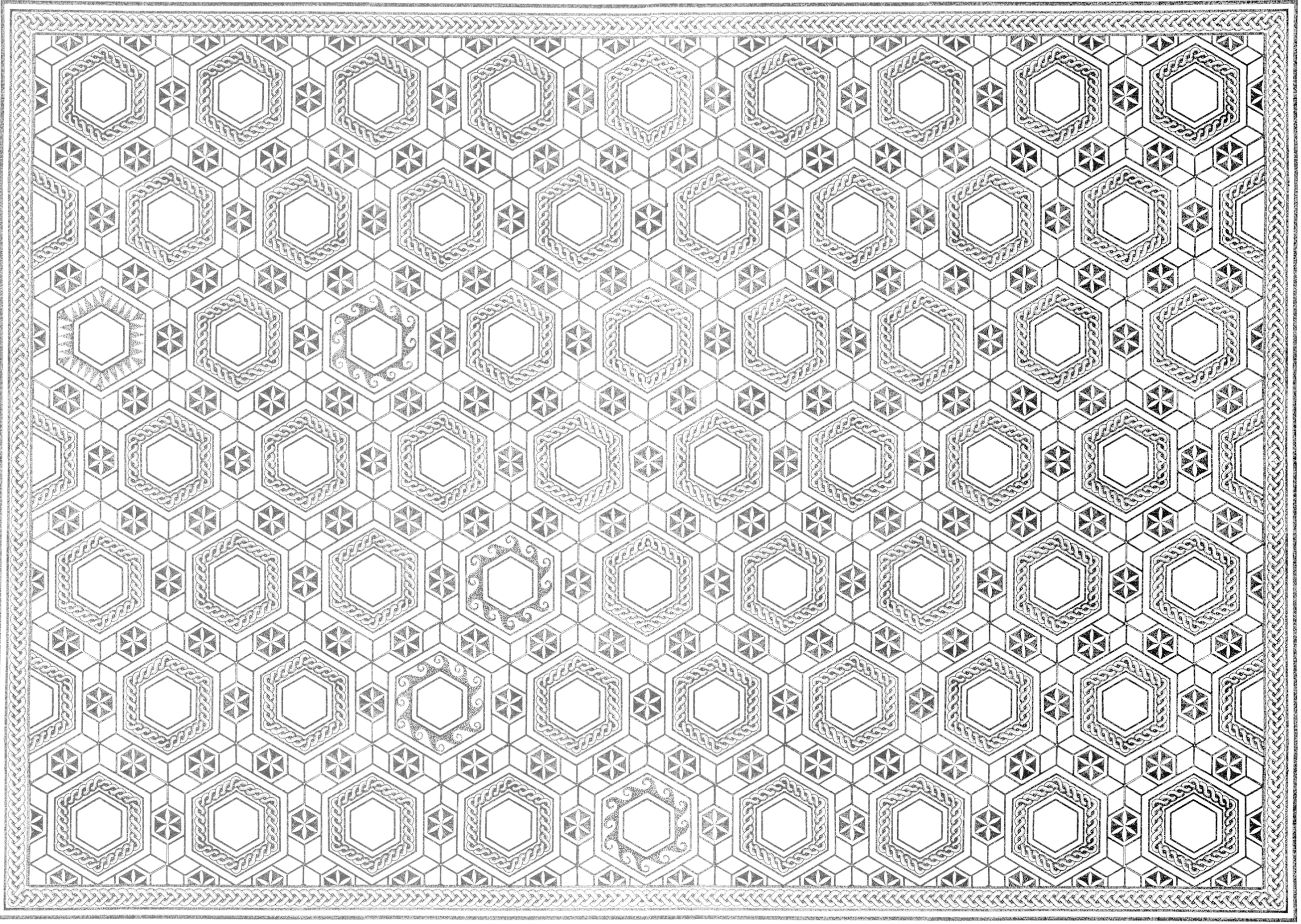




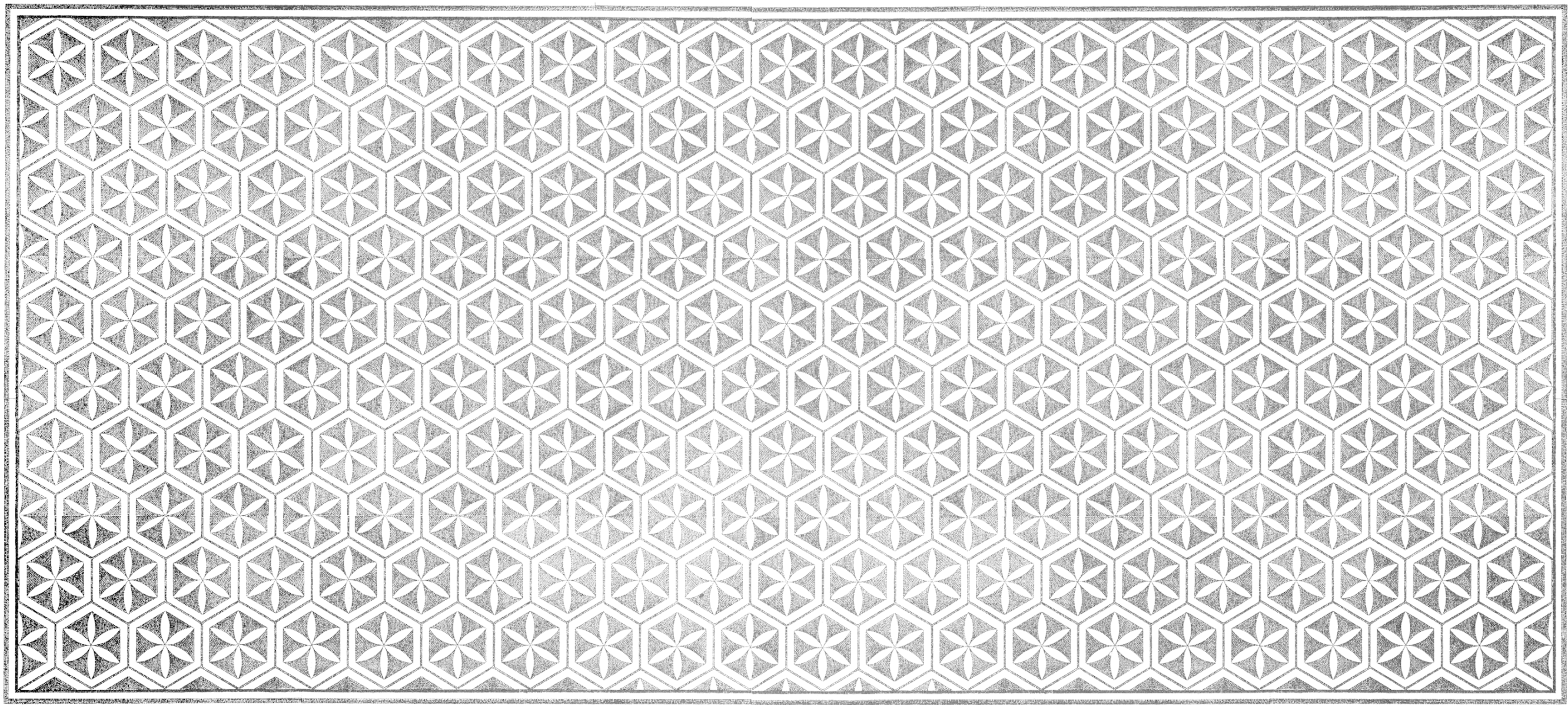

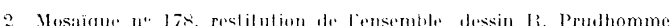




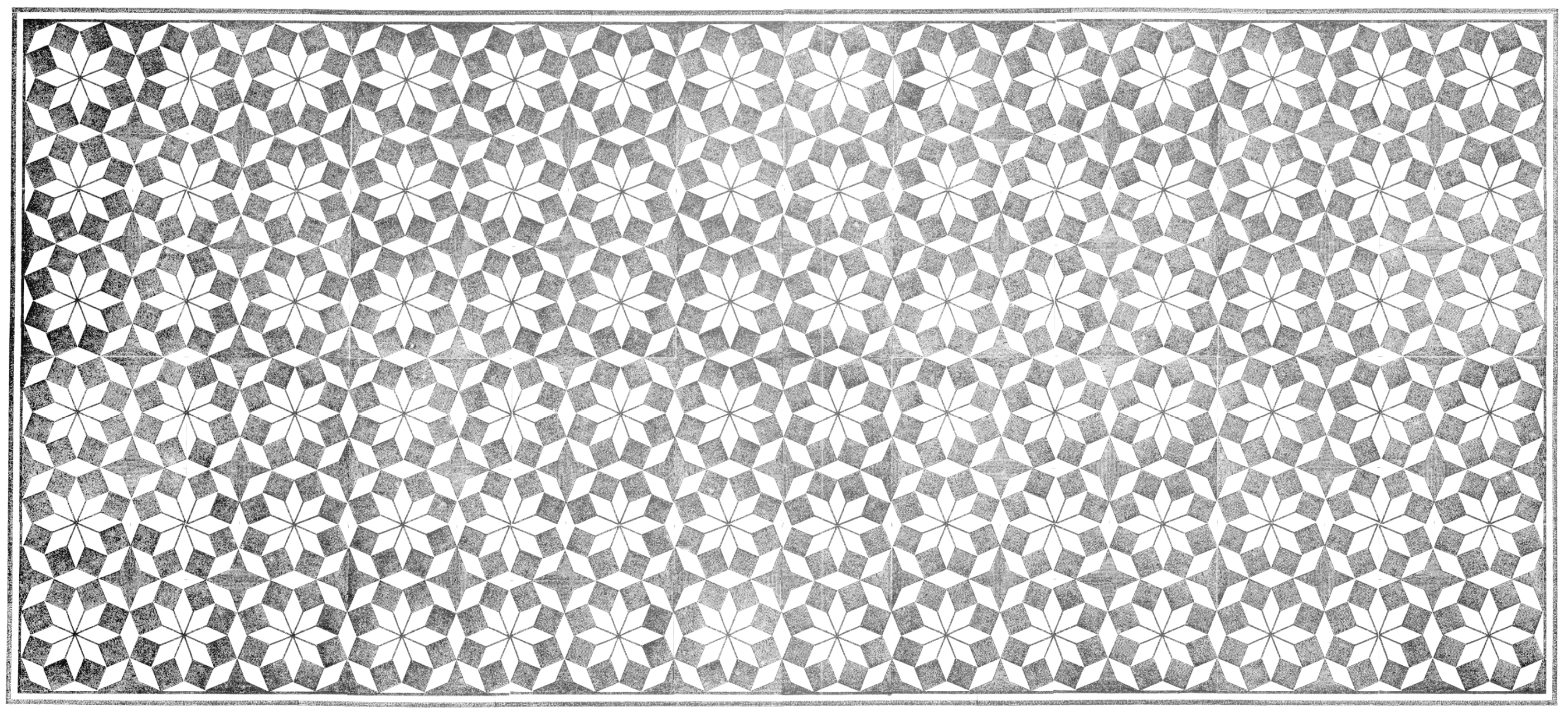


assemblage serré ont permis de tracer tous les dessins avec une netteté parfaite ». Affirmation exacte pour le dessin de la trame elle-même, mais qui laisse malheureusement à désirer en ce qui concerne le détail des remplissages. Là, le dessinateur s'est contenté de figurer des motil's vaguement circulaires ou rectilignes, à peine discernables. Tuutefois, le second dessin (fig. 10) de la mosaïque en reproduit un angle : on y voit un hexagone comportant une bordure interne en tresse à deux brins polychromes et, au centre, un dauphin bleu et vert qui s'enroule autour d'un trident. Nous devons nous satisfaire, pour les autres hexagones, des mentions suivantes : "poissons, vases, oiseaux 》, et, dans un autre texte, "une poule d'eau ».

Les dimensions des motifs et les mesures globales permettent une restitution sûre pour le canevas d'ensemble $(9,50 \mathrm{~m} \times 7 \mathrm{~m}$; côté des grands hexagones : $39,5 \mathrm{~cm}$; côté des petits hexagones : $11,5 \mathrm{~cm})$. C'est une composition en nids d'abeilles déterminés par des bandes d'étoiles de six losanges et de petits hexagones adjacents, chacun de ces derniers étant chargé d'un six-feuilles blanc sur fond noir. Ce pavement d'une très grande surface comportait soixante-trois hexagones complets et, à la ligne de chute du canevas, trois demi-hexagones de chaque côté. La bordure entourant le tapis est une tresse à trois brins polychromes (larg. : $20 \mathrm{~cm}$ ). La seule incertitude (outre celle des remplissages figurés) porte sur les bordures internes des grands hexagones. Pour un d'entre eux, on distingue nettement qu'il s'agit du motif bien connu des «dents de loup », dont la ligne est interrompue à chaque angle de l'hexagone, par une "pointe" en fer de lance. Pour tous les autres hexagones, on hésite entre la bordure de postes simples ou la tresse à deux brins. Dans l'ignorance de la répartition de ces deux types, nous avons préféré la bordure en tresse qui est la plus fréquente, mais il est probable (comme nous l'apprennent les autres exemples de pavements de la région construits sur ce thème) que les bordures alternaient selon des rythmes réguliers pour éviter la monotonie.

La datation que l'on peut proposer pour cette mosaïque ne peut s'appuyer évidemment que sur une mise en série stylistique. L'histoire de la trame est relativement bien connue et les traits caractéristiques des remplissages orientent immédiatement vers les œuvres des ateliers de Vienne. H. Stern ${ }^{28}$ et, à sa suite, Janine Lancha ${ }^{29}$ en ont retracé l'évolution et nous les suivons dans les grandes lignes de leur analyse, sauf pour ce qui est des datations, sur lesquelles il faut revenir. Selon Janine Lancha, le canevas a été créé en Italie du Nord, peut-être à Aquiléc au ${ }^{\mathrm{er}}$ s. ap. J.-C., puis s'est transmis à la Gaule, principalement à Vienne, qui l'a mis à la mode avec un grand brio, en l'enrichissant par un répertoire ornemental polychrome d'une exceptionnelle richesse au cours du $\mathrm{II}^{\mathrm{e}} \mathrm{s}$. et jusqu'au premier quart du $\operatorname{III}^{\mathrm{e}} \mathrm{s}$. Évolution exacte dans l'ensemble, mais qu'il convient de discuter pour déterminer dans quelle phase la mosaïque d'Anse peut trouver sa place.

Il est probable, en effet, que les deux exemples les plus anciens sont fournis par Aquilée au $\mathrm{I}^{\text {er }}$ s. de notre ère ${ }^{30}$, ainsi que par Cividale del Friuli ${ }^{31}$. L'austérité des remplissages

28 H. Strern, Mosaïques de la région de Vienne (Isère), dans Gallia, 29, 1971, 1, p. 135-137.

29 Janine I.мхсия, Mosaïques géométriques, les aleliers de Vienne (Isìre), Rome, 1977, p. 59-65.

30 On en connaît deux exemples à Aquilće: l'un, au Fondo Cossar, est traité uniquement en noir el blanc, avec des crateres dans les hexagones; l'autre, provient du Fondo C.A.L. : les hexagones sont chargés d'une étoile à six pointes, frappéc elle-même d'un petit hexagone à six-feuilles noires.

31 Janine I.avchs, op. cit., fig. 25. 
géométriques y prédomine : les grands hexagones sont chargés d'étoiles à six branches, les petits hexagones et les étoiles de huit losanges sont tantôt ajourés, tantôt simplement blancs. Les bordures internes, ou n'existent pas, ou sont uniquement traitées dans le type le plus ancien, les postes. On peul s'arrêter aussi sur un troisième exemple récemment signalé ${ }^{32}$ à Rimini (mosaïque dite du "Mercato coperto") dans laquelle les hexagones semblaient bien comporter des scènes figurées mais traitées encore en noir et blanc comme l'ensemble du pavement. Une datation vers la première moitié du $\mathrm{II}^{\mathrm{e}} \mathrm{s}$. lui est assignée. Dans la seconde moitié du siècle apparâll la couleur avec un sccond excmple à Rimini ${ }^{33}$ (mosaïque du Palais Gioia), et la somptueuse mosaïque de Lycurgue et Ambrosic à Aquilée ${ }^{34}$. Elles se situent à la même période et sont du même niveau artistique : polychromic éclatante des remplissages figurés, variété des motifs ornementaux qui cnvahissent tous les espaces du schéma, l'aspect géométrique du fond en noir et blanc ayant été complètement oblitéré.

Les excmples de la Gaule en dérivent directement, mais ne sont pas de pâles copies. Toute la série viennoise se dislingue, au contraire, par son originalité. On constate, d'une part, une grande vivacité dans le répertoire ornemental des hexagones, chargés de fleurons, d'oiseaux, de vases, de petits objets chers au style viennois, et, de l'autre, une rigueur et une sécheresse constantes dans les motifs secondaires (losanges des étoiles, petits hexagones) pour la plupart dépourvus de remplissages et traités en noir et blanc, ce qui donne au pavement un bel équilibre. La mosaïque d'Anse apparticnt indiscutablement à cette série. Citons le $n^{0} 338$ de Vienne ${ }^{35}$ où l'on a le même réseau avec étoiles blanches et petits hexagones frappés d'un six-feuilles blanc sur fond noir, contrastant avec des fleurons, des touffes de verdure, des canards et des vases qui ornent l'intéricur des hexagones. Comme à Anse, les bordures internes sont en tresse à deux brins, en postes, et en lignes de dents de loup. Ce pavement est daté par l'aulcur du fascicule consacré à Vienne, des "années 175-200, sa version ćtant plus composite " que celle des autres représentants de la série viennoise. L'argument n'est guère convaincant, et si l'on examine les exemples analogues, on saisit bien des différences de surcharge et de qualité dans les remplissages, mais il ne paraît pas évident d'en faire un critère de chronologie relative très assuré. La mosaïque Diederichs (le numéro 358 du Recueil) est sans doute de la même main que la mosaïque "Grange * (numéro 375), car les vases et les fleurons qui la décorent sont singulièrement proches, mais doit-on accepter la datation de 150-175, qui n'est étayéc sur aucun argument ? Quant au numéro 396 (Saint-Romain-en-Gal), véritable feu d'artifice de fleurons de fantaisie, de motifs rayonnants, éclatés, à plusieurs cercles concentriques, on no saurait dire pour autant qu'il est le plus tardif de la série, du "premier quart du $\operatorname{III}^{\mathrm{e}} \mathrm{s}$. ». Sa proximité avec une autre mosaïque de la domus (laquelle n'est pas datée par des arguments archéologiques précis) ne saurait avoir valeur d'argument. Signalons, enfin, une mosäque récemment

32 G. Rrcciont, Rimini, Mosaici pavimentali del I et II secolo d.c. con motivi figurali, dans $111^{\circ}$ Colloquio internazionale sul mosaico antico (Ravenne, sept. 1980), Ravenne, 1983, p. 23-34, fig. 11-12.

33 Ibid., pl. couleurs, p. 148.

34 L. Bertacciu, Architetlura e mosuicn, dans I) Aquileia a Venezia, Milan, 1980, p. 165-166 el pl. couleurs 136-138.

35 Janine Lancin, Recueil général des mosaiques de la Gaule, III, 2, Vienne, $X^{\mathrm{e}}$ supplémenl à Gallia, $\mathrm{n}^{0} 338$, pl. XC. 
étudiée ${ }^{36}$, mise au jour à Vienne lors de la construction immobilière dite des "Nymphéas 》. J. Lancha remarque finement que l'on trouve dans cette mosaïque, et pour la première fois à Vienne (ce qui est significatif sur un total de 212 notices consacrées à cette ville), un trait caractéristique des ateliers de la Basse Provence : la tresse pointillée, c'est-à-dire détachéc sur un fond noir avec, à chaque croisement des brins, une tesselle blanche posée sur la pointe. Nous avons eu l'occasion ${ }^{37}$ de proposer de voir dans ce détail une particularité des ateliers provençaux du I $^{\text {er }}$ s., qui disparaît, nous semble-t-il, vers 150. Faut-il penser qu'il s'agit là, à Vienne, d'un trait d' archaïsme», et faut-il pour cette raison placer cette mosaïque "plutôt dans le premier quart du iI ${ }^{\mathrm{e}} \mathrm{s}$. "? Nous ne pensons pas qu'il faille remonter à ce point la date de ce pavement. A nos yeux, la tresse pointillée disparaissant vers 150 , nous sommes au moment charnière du milieu du Ir $^{\mathrm{e}}$ s., où l'École de Vienne va prendre son essor et acquérir son originalité. Le mosaïste qui a emprunté ce "tic d'écriture » aux ateliers du Sud de la Provence, est encore soumis au style un peu sec du I $^{\mathrm{er}}$ s., hérité du répertoire italique, et conservé presque tel dans le sud de la "Province ». Au lieu des fleurons déliés, inventifs, du "vocabulaire " viennois, il forme des motifs symétriques, raides et il a même recours au vieux poncif du nœud de Salomon. Nous avons peut-être là, effectivement, le premier exemple de la série gallo-romaine, mais la date de 150 ap. J.-G. nous paraît mieux lui convenir. Et comme nous disposons pour cette domus d'un précieux lerminus posl quem offert par l'abandon de la maison "avant la fin du $\mathrm{II}^{\mathrm{e}} \mathrm{s} . \|^{38}$, nous pensons qu'il est légitime d'inscrire tous les exemples de Vienne cités ci-dessus entre le milieu et la fin du II e s., sans chercher, dans l'état actuel de nos connaissances, à les classer chronologiquement.

Les autres mosaïques construites sur ce schéma sont toutes étroitement tributaires de l'influence viennoise. Cela est clair pour les plus proches, comme le beau pavement de Limony dans l'Ardèche ${ }^{39}$ où nous retrouvons les trois types de bordures présents à Anse, mais aussi pour le numéro 99 de Lyon $^{40}$ ou encore le fragment de Penol (Isère) ${ }^{41}$, et même, plus au s., pour la mosaïque d'Auriol ${ }^{42}$ dont les fleurons sont de style viennois. A ces quatre exemples, la deuxième moitié du II $^{\mathrm{e}} \mathrm{s}$. pourrait également convenir.

Plus frappants encore, à cause de leur éloignement de la capitale des Allobroges, sont les deux cas de Périgueux. Pour la première fois dans le Sud-Ouest, nous avons la preuve incontestable d'une adoption parfaite de la "manière viennoise ». On connaissait de longue date le pavement du quartier de la Tour de Vésone $e^{43}$, mais les fouilles

36 Janine Lancha, Découverles archéologiques récentes à Vienne (Isère), dans Monuments Piot, 64, 1981, p. 94102 , fig. $56-58$

37 H. Lavagine, Recueil général des mosäques de la Gaule, Narbonnaise, III, I, X $X^{\mathrm{e}}$ supplément à Gallia, 1979, p. 20.

38 Janine L.aNcira, op. cil., p. 58.

39 Figure 9 dans Gallia, 33, 1975, p. 533 et dans .1. Guigal, Deux siles gallo-romains en Haut-Vivarais: Limony et Talencieux, dans Archeologia, 112, 1977, p. 60-63.

40 II. STren, Recueil général des mosaïques de la Ciaule, Lyonnaise, II, $1, X^{\mathrm{e}}$ Supplèment à Gallia, no 99.

41 iallia, 16, 1958, p. 382, fig. 6.

42 G. LAFAYs, Invenlaire des mosaïques de la (iaule, I, 1909, $\mathrm{n}^{0.3} 63$ et 64 . Cf. l'aquarelle de N. Coste, dans Mosaĭques, recueil factice, Bibliolheque Nationale, G.b., 2 a, pl. I.

43 Invenlaire des mosaïques de la lianle, ibid., $\mathrm{n}^{\circ} 560 \mathrm{~A}$. 
viennent d'en révéler un second ${ }^{44}$ très proche par son style et que l'on n'aura pas de peine à attribuer, sinon à une équipe itinérante venue de Vienne, du moins à des artistes formés à Vienne. Le premier exemple a été opportunément réétudié par J. L. Tobie qui y voit très justement la mosaïque pavant le sanctuaire de Cybèle ${ }^{45}$. Les dimensions sont presque celles d'Anse $(10,50 \mathrm{~m} \times 7,45 \mathrm{~m})$ et la différence tient surtout à l'iconographie, nécessairement ici plus contraignante puisqu'il s'agit d'un templc. L'auteur a fait remarquer que la présence du culte de Cybèle à Périgueux s'explique aisément par les liens bien attestés, non seulement sur le plan des routes et des voies commerciales, mais aussi sur celui des élites dirigeantes que nous fait connaître l'épigraphic, entre les cités de Lyon, de Vienne et la capitale des Petrocorii. Nous ajoutcrions volontiers, pour notre part, que ces mêmes liens permettent de comprendre également la résurgence de ces compositions si typiques de la moyenne vallée du Rhône dans cette région de l'Aquitaine où elles surprennent au premier abord ${ }^{46}$.

Nous ne ferons pas entrer dans cette revue la mosaïque de Condat-sur-Vienne (Haute-Vienne) $)^{47}$, pourtant bâtie sur le même canevas, parce qu'elle participe du répcrtoire du "décor multiple », lequel n'est pas présent dans le cas d'Anse, ni non plus les exemples d'Ouzouër-sur-Trézée (Loiret) ${ }^{48}$, dont l'ordonnance ménage un emblema central dans un esprit tout autre qu'à Anse. Peut-être appartiennent-ils, eu égard à cette modification fondamentale, à l'époque sévérienne.

Ces approximations chronologiques nous permettent de caractériser micux la mosaïque qui nous occupe : sa trame comme son style en font une mosaïque "viennoise " que l'on peut situer dans la seconde moitié du $\mathrm{II}^{\mathrm{e}} \mathrm{s}$.

Mosaïque $n^{\circ} 1^{17} 8$ (fig. 12).

Le second pavement offre moins d'intérêt, car son décor est peu caractéristique. C'est, à nouveau, une composition en "nids d'abeilles", mais ceux-ci sont simplement formés par la juxtaposition d'hexagones adjacents timbrés chacun d'un six-feuilles blanc sur fond noir. Schéma assez ordinaire, sa voguc n'a pas été très répandue. L'Italie en donne quelques exemples, à Este ${ }^{49}$, à Ancônc ${ }^{50}$, ou encore à Vérone ${ }^{51}$ dans une mosäque où l'on a

44 Gallia, 41, 1983, 2, p. 449, fig. 5.

45 J.-L. Товге, Une nouvelle définilion du quartier antique de la Tour de Vésone, dans Périguetti et le Périgord, Fédération IItstorique du Sud-Ouest, Actes du $X X X^{\mathrm{e}}$ Congrès d'Éludes régionales de Périgueux (avril 1978), 1981, p. $42-51$, fig. p. 46.

46 Nous omettons de parler ici de la mosaique de Montmorot (Recueil général des mosaíques de la (iaule, II, 3, $n^{\circ} 468$ ), datée "fin II $^{\mathrm{e}}$ ou début rme s. ", qui est d'un style assez difiérent.

47 Cf. fig. 16, p. 64 dans l'étude d'H. Stenn, Mosaïques de Pontchevron près d'Ouzouër-sur-Trézée, dans Gallia, $25,1967$.

48 J.-P. Darmon, Recueil général des mosaïques de la Gaule, Lyjonnaise, II, 3, X ${ }^{\mathrm{e}}$ Supplément à Gallia, $\mathrm{n}^{\circ}$ 468, le dale, avec quelques réticence, de l'époque sévérienne.

49 Este, dans Not. Scav., 1941, p. 61, fig. 18 (les hexagones sont ici dans un grand cercle, mais également frappés d'un six-feuilles;.

50 Ancône, dans Not. Scav., 1926, p. 379, fig. 1. Le pavement est d'autant plus intéressanl à comparer avec celui d'Anse, que dans le tapis central, on a une étoile de huit losanges cantonnés de petits carrés sur la pointe el, dans deux rallonges symétriques, le mosaiste a choisi le motif des hexagones tangents chargès d'un six-feuilles, comme à Anse.

51 Vérone, dans Not Scav., 1965, p. 48, fig. 20. 
voulu rompre la monotonic des remplissages en faisant alterner les lignes de six-feuilles avec des hederae. On peut également retenir des exemples d'Aquilée ${ }^{52}$, dont l'importance comme centre de création de motifs qui se diffusent en Italie du Nord puis, de là gagnent la Gaule, est de mieux en mieux attestée ${ }^{53}$. Ce qui frappe à Anse, c'est l'utilisation de ce canevas extrêmement répétitif, sous sa forme la moins variée, dans une mosaïque ayant une surface aussi importante (presque $43 \mathrm{~m}^{2}$ ). Aucun détail de style ne peut fournir d'éléments de datation, mais la période retenue pour le pavement précédent, la deuxième moitié du $\mathrm{II}^{\mathrm{e}} \mathrm{s}$., peut également lui convenir.

Mosaïque no 177 (fig. 13).

Ce n'est pas la banale composition en étoiles de huit losanges, petits carrés droits et grands carrés sur la pointe, mais une trame beaucoup plus rare, et même, à notre connaissance, unique en Gaule, au moins sous cette forme. Elle repose sur le motif des étoiles de huit losanges (ici blancs), mais les extrémités sont cantonnées de huit petits carrés noirs sur la pointe et déterminent des losanges et de petits octogones, ici chargés d'étoiles à quatre branches noires. La rareté du schéma tient à la rigueur avec laquelle les deux «motifs organisateurs" sont traités : étoiles et octogones sont situés exactement au même niveau visuel et s'équilibrent parfaitement sans qu'aucun remplissage ne vienne renforcer l'importance de l'un au détriment de l'autre. La variante la plus répandue n'a pas été retenue $e^{54}$ : elle fait apparaitre de grands octogones, formés par les étoiles de huit losanges les petits carrés et les losanges blancs qui les entourent, en les séparant nettement par de grands carrés, qui se prêtent à des remplissages variés. Par rapport aux nombreuses variantes de compositions en étoiles de huit losanges, celle qui a été choisie ici est assurément la moins banale et la plus austère par sa bichromie. Une fois encore, c'est son extension à une surface aussi considérable qui retient l'attention. Dans l'exemple d'Ancône cité plus haut ${ }^{55}$, on observe une utilisation semblable de l'ćtoile de huit losanges cantonnés de petits carrés sur la pointe, mais en tant que motif central pour le tapis, et non pas répété, comme à Anse, 84 fois ! L'absence d'éléments de comparaison rend la datation difficile, mais on serait tenté de faire le rapprochement avec des mosaïques noires et blanches d'Ostie (par exemple, celles de la Casa di Apuleio) ${ }^{56}$, qui présentent ce genre de trames monotones, vers le milicu du II $^{\mathrm{e}}$ s., et dans lesquelles les mêmes motifs sont repris dans des tapis couvrants sans aucune recherche de variété.

52 Aquilée, dans Boll. d'Arle, 49, 1964, fig. 6 (Casa del Corridoio). On note que la mosaíque décore un corridor, auquel son caractère répétitif se prête assez bien. C'étail peul-être aussi le cas à Anse. Autre exemple, ibid., p. 260, fig. 70 (daté du I $^{\mathrm{er}}$ s. ap. J.-C.).

53 M. Donderer, Cullura aquileiese in mosaici geomelrici romani dell'Occidente, dans Antichità Altoadriatiche, 19, 1981, p. 225-238, et Beobachlungen zum Stil der römischen Mosailien Nordilaliens in favischer Zeit, dans Colloque Ravenne, 1983 (cil. n. 32), p. 39-50.

54 Un exemple particulièrement significatif à Cyyrène, cf. P. Mıngazzın , L'Insula di Giasone Magno à Cirene, dans Monografia di Archeologia Libica, 8, 1966, pl. XI, 4.

55 Voir supra, note 50.

56 G. BeCatTI, Oslia, IV, mosaici e pavimenli marmorei, liome, 1961, pl. XXXVII. 
Le rapport de Chenavard et Rey indique que ces trois mosäques étaient dans un état parfait de conservation lors de leur dégagement. On doit donc se demander quel a été leur sort ultéricurement. Probablement «détruites», selon P.-M. D)uval et J. Guey ${ }^{57}$, "détruites par le cultivateur", pour H. Stern et Michèle Blanchard-Lemée $e^{58}$, peu après leur découverte. Il n'en n'est rien, et un texte méconnu de l'Almanach du Beaujolais ${ }^{59}$ paru cn 1941, nous indique que ces mosaïques étaient encore en place à cette époque. Un marbrier-sculpteur de Villefranche-sur-Saône, M. Bornarel, relate comment il reçut la mission de les déposer et de les convoyer chez un ccrtain Comte de Val, à Paris, qui les avail achetées. L'artisan, qui n'ignore pas qu'elles avaient été déjà mises au jour presque cent ans avant lui, les décrit à peu près dans les mêmes termes que ses prédécesseurs de 1845. Il ajoute simplement dans les motifs de remplissages des hexagones de la mosaïque $n^{0} 176$, "des cornes " (entendre des rhytons), "une poule d'eau ", des "poissons", ce qui précise et confirme une nouvelle fois l'appartenance de ce décor au répertoire ornemental de la production de Vienne et de Lyon. Bornarel explique, en outre, la méthode de dépose qu'il a pratiquéc avec succès : après avoir chauffé le tessellatum, il en a encollé la surface avec de la toile, puis a coulé une couche de ciment de $2,5 \mathrm{~cm}$ sur cette toile en insérant dans le ciment des « lattes » de bois pour rendre le support plus rigide. Par chance, le nucleus étant peu adhérent ${ }^{60}$, l'opération de désolidarisation s'est faite sans difficulté, et unc fois la dépose exécutée, il a coulé un second lit de ciment, de $6 \mathrm{~cm}$ d'épaisseur, sur la face arrière du tessellatum. Le transport à Paris a donc pu se réaliser, et il est probable que ces mosaïques s'y trouvent encore, dans quelque hôtel particulier que nous n'avons pas encore pu retrouver.

Ces trois mosaïques complètent done de manière essentielle notre connaissance du décor pavimentaire de la Grange-du-Bief, en même temps qu'elles nous aident à préciser le plan de l'ensemble des bâtiments. Elles confirment les observations d'H. Stcrn et de Michèle Blanchard-Lemée ${ }^{61}$ sur l'influence des ateliers viennois dont on sent la trace indéniable sur les mosaïques d'Anse, mais elles engagent à remettre en cause la datation sévérienne que ces auteurs avaient adoptée. Celle-ci nous est apparue trop tardive et nous proposons de lui substituer la deuxième moitié du II $^{e}$ s. Il semble bien, d'ailleurs, que dans toute la région lyonnaise, la fin du $\mathrm{II}^{\mathrm{e}}$ s., en liaison avec l'incendic de 197, ait été marquée par des destructions importantes dans l'habitat rural ${ }^{62}$. Cette nouvelle datation ne modifie

57 J. Guey et P.-M. Duval, op. cil., p. 108.

58 Recueil des mosaïques de la Gaule, II, 2, p. 35 el 40.

59 Il nous a été aimablement communiqué par M. Yvon Tenu.

60 Sur ces problèmes, cf. nolamment Cl. BAssier, Acles du $11 I^{\mathrm{C}}$ Symposium inlernational sur la Conservation des mosaiques, Aquilée (3-7 octobre 1983) à paraître, el II. LavaGNr, Les maïtres-mosaïstes dans Bulletin Monumenlal, 1984 , t. 142,1984, p. 305-316.

61 Recueil cil., II, 2, p. 34. On comparera aussi avec la mosaïue tris proche par son style, de Bourbonl'Archambaull (fig. 14), récemment republiée par J. (on l'Allier, dans Rev. Arch. Siles, 23, 1984, p. 26.

62 Cf. SL. Whlker, L'archéologie rurale dans les départements du Rhône el de la Loire: civilas des Ségusiaves et colonie de Lyon, dans Recherches récentes en archéologie gallo-romaine et paléochrétienne sur Lyon et sa région, Brilish 


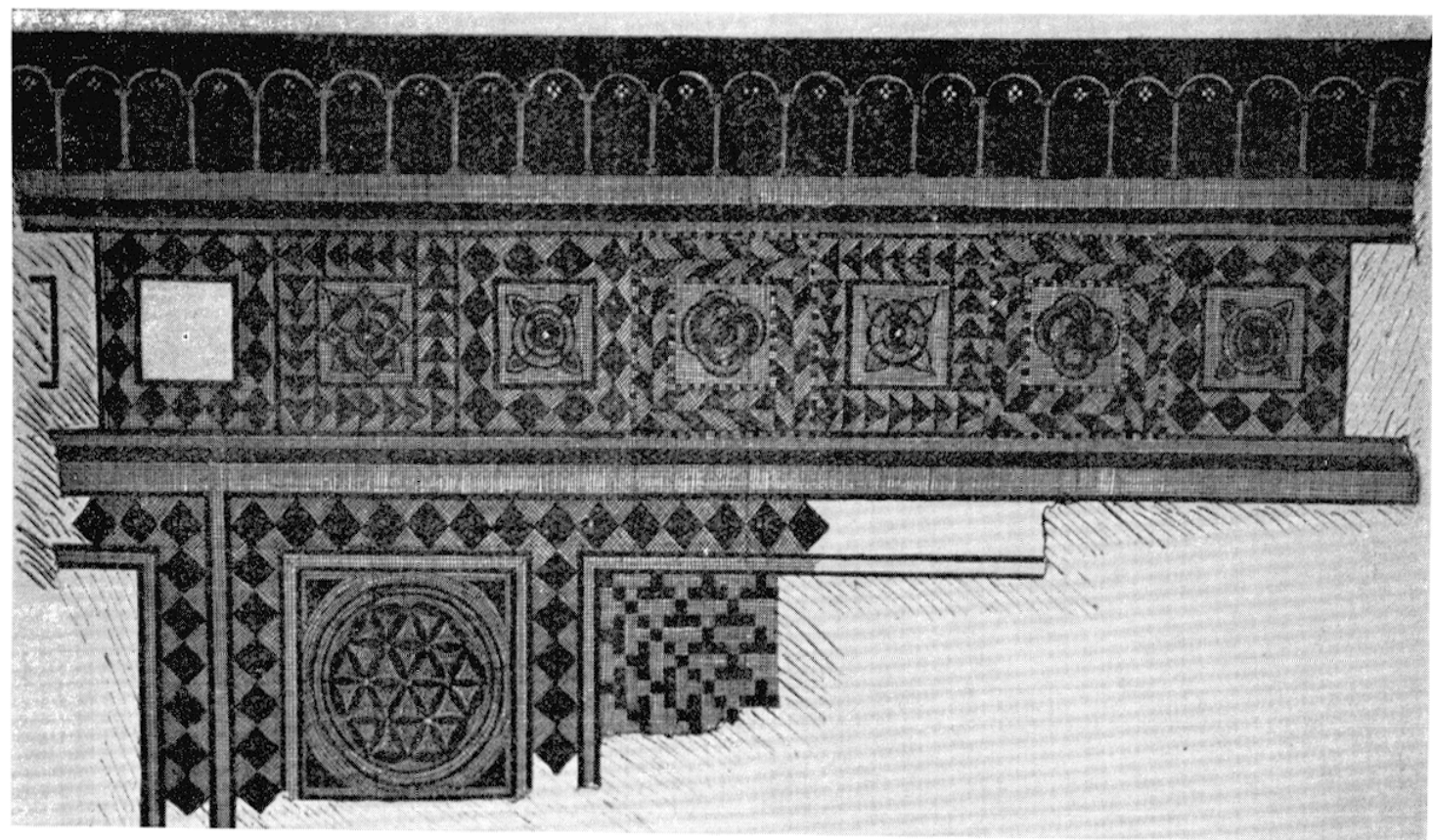

14 Mosaïgue de Bourbon l'Archambault. (Cliché J. Corrocher.)

en rien celle qui avait été assignée par P.-M. Duval et J. Guey en 1960 pour la mosaïque aux proues de navires qui orne aujourd'hui le Musée du château à Anse. La première moitié du $\mathrm{II}^{\mathrm{e}} \mathrm{s}$. qu'ils suggéraient comme date, à la fin de leur étude, nous paraît toujours valable, car les détails iconographiques de ce pavement ${ }^{63}$, comme sa trame de construction, nous paraissent devoir la placer à une époque sensiblement plus haute que celle que nous attribuons aux trois mosaïques de 1845 . Il en résulte donc que l'établissement du décor des sols à Anse a dû s'effectuer en deux phases : dans la première entre 100 et 150 ap. J.-C., le grand oecus principal de la demeure a reçu son pavement aux proues de navires. (ie motif de navalia unique en Gaule, et rare en Italie, ne nous semble pas purement décoratif. L'hypothèse qu'il soit comme l'emblème de la profession du commanditaire, un armateur, un propriétaire d'une flotille de bateaux de commerce sur la Saône et l'Azergues ${ }^{64}$ nous

Archaeological Reports, Int. Series, 108, 1981, passim, notamment p. 321. Nous altirons également l'attention sur une hypolhèse de IR. Perraud (dans Aclivités Beaujolaises, 22, 1965, p. 34) qui rappelle la trouvaille, en 1840, à Trévoux, d'un trésor de 10000 deniers d'Albin (legrende : Concordia militum). L'auteur émet l'hypothèse que ces monnaies, qui n'avaient jamais circulé, devaient servir à la solde des légione d'Albin et qu'après la défaite de celui-ci par SeptimeSévère en 197, le payeur aux armés aurait, enfoui le lrésor pour éviter qu'il ne tombât aux mains de l'ennemi. Ce qui laisse supposer dans la campagne lyonnaise des batailles ou des coups de mains entre les armées rivales, dont les riches villas des notables eurent sans doute à pâtir.

63 A l'exemple du pavement aux proues de navires de la Via Ardeatina à Rome (conservée au Musée du Vatican) et datée d'époque républicaine (M. I. Morricons Matıni, Mosaici romani a casseltoni del I Secolo a.c., dans Archeologia Classica, 17, 1, 1965, p. 79 et sq.) on ajoutera la mosaïque de Tricario (V. de Cicco, dans Not. Scav., 1903, p. 350) el une autre, perdue, d'Ostie (Brecarti, op. cil., p. 234). Autres exemples : à Lanuvium (collégiale Sainte-Marie Majeure) (cf. Nol. Sicav., 1953, p. 315, fig. 6) el à Pompéi, dans la Casa di Popidius Priscus (G. Fı) res.lı, Gli scavi di Pompei dal 1861 al 1872 , Naples, 1873, p. 151). Je motif est trate d'une maniere également tres proche sur une peinture

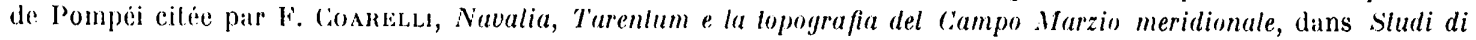
lopografia anlica dell liniversila di lioma, 1968, p. 27-37.

61 Celte hypothese de P.-M. Juval et J. Guey a éte constamment reprise par la suite, notamment par 
paraît séduisante. Puis, dans une deuxième phase, entre 150 et 200 , on peut imaginer que son affaire ayant pris une certaine extension, les bâtiments auraient été développés vers l'o. et auraient reçu alors un nouveau décor de mosaïques, les pavements nos $176,177,178,179$, ainsi que les nos 182 et 183 qui pavent les deux pièces situćes en retour du côté s., dégagées par R. Perraud en 1964-1966. Ce dernier proposait déjà cette date en signalant ${ }^{65}$ que les céramiques trouvées en remplissage sous les pavements des pièces VII et VII b se situaient dans la deuxième partie du $\mathrm{II}^{\mathrm{e}} \mathrm{s}$.

L'ensemble des données de fouilles depuis 1843 jusqu'à nos jours, permet d'établir un plan (fig. 2) attestant l'existence de plus de vingt pièces, sans compter les dix salles portées sur le plan de Chenavard-Rey, dont les sondages ultérieurs devront encore s'employer à vérifier la présence. On compte désormais treize mosaïques assurées et deux autres simplement probables ${ }^{66}$. Par rapport au dernier plan en date, celui de St. Walker ${ }^{67}$, les substructions connues sur le site ont plus que doublé. Pcut-on, à partir de ces nouvelles données, substituer à l'appellation de villa celle d'un vicus ? Outre les difficultés à établir clairement les critères de définition d'un vicus ${ }^{68}$, nous pensons que la réponse est prématurée. Malgré certaines anomalies, le plan que nous présentons rattache la disposition des bâtiments à la série des villas à cour avec une galerie de façade à colonnade, qui serait complétée par des constructions annexes étagées sur les différentes terrasses de la colline. Dans l'état actuel de nos connaissances, il nous paraît plus probable de penser, sclon un schéma souvent confirmé par les études de R. Agache ${ }^{69}$ que le vicus fortifié d'Anse (la "forteresse postale» de C. Jullian) s'est développé à quelques kilomètres du grand domaine et de la villa, qui demeure, en tout cas, l'une des plus importantes de la Gaule du Centre.

\section{H. LAVAGNE}

St. WALKer, La campagne lyonnaise du Ier s. av. J.-C. jusqu'au $V^{\mathrm{e}}$ s. après J.-C. ibid., p. 309. On comparera également avec la puissance de ces grands patrons d'entreprises de batellerie, mise en valeur pour les pays de la Loire par G. Ch. PICARI), Ostie et la Gaule de l'Ouest, dans BSNAF, 1980-1981, p. 103-104 et MEFRA, 93, 1981, 2, p. 883-915. Toutefois, l'affirmation de Charles Picard (Sur un portique décoré avec des élemenls de proues de navires en bordure de la mosaique de la Grange-du-Bief, Rhône, dans Rev. Arch., 1963, p. 112-114) pour qui ce serail un motif dérivé des trophées hellénistiques reste solidement argumentée.

65 R. Prrraud, dans La Physiophile, cit., p. 32 et 39-40.

66 Les numéros 181 et 185 du Recueil général des mosaïques de la Gaule, II Lyonnaise-2, X' supplémenl à Gallia. Notons pour se $n^{\circ} 185$ que la tradition locale recueillie par P.-M. Duval et J. GuEy dans leur article (op. cit., p. 88, n. 25) selon laquelle une mosaïque d'Anse aurail été achetée par le Prince Napoléon, a loutes chances d'être exacte. En effet, on sait que le Prince collectionnait les antiques pour la "villa pompéienne " qu'il avait fait construire à Paris, avenue Montaigne (cf. M.-Cl. Dejean de la Batie, La maison pompéienne du Prince Napoléon, dans (iazelle des Beaux-Arts, 1976, I, p. 127-I34 et M. N. de GARY, La maison pompé̈enne du Prince Napoléon, calalogue de l'exposition du Musée des Arls Décoratifs, Paris, 25 avril-l er octobre 1979), Les œuvres d'arl furenl vendues le 23 mars 1868 et Je catalogue de vente rédigé par W. Frä̈NrR (Les anliques de la Colleclion du Prince Napoléon Jérôme, Paris, 1868) porle sous le numéro 295 la mention d'une table en mosaïque de forme octogonale, avec des ornements polychromes. Malgré l'absence de provenance, il pourrait bien s'agir d'un fragment de mosaïque d'Anse. On sait que le restauratcur, Cl. Mora, fit une lable en mosaiqque à partir de fragments de la mosaïque du Musée (actuellement à la Mairie d'Anse). Ce genre de montage n'aurait rien de surprenant sous le Second Empire. Il est possible que cette mosaique ail fait partie des objets récupérés par l'architecte qui avait conçu la maison pompéienne, Charles Normant. Celui-ci les regroupa en un muscie provisoire, à l'Hôtel Sully, 62, rue Saint-Antoine à Paris (cf. Le SEnne, Notes sur la villa pompeienne de l'avenue Montaigne dans Bull. Soc. Hist. Arch. des VIII' et XVII arrondissements, 1912, p. 77-85). Mais cette collection fut également dispersée par la suite (Ch. Nonmanr, Les princières reliques, dans Mélanges Le Senne, ibid., 1915-1916, p. 187-194). Nous n'avons pu les retrouver.

67 Op. cil., p. 296, fig. 5.

68 Cf. les réflexions de Sl. WaLkER, p. 321 et $s q$.

69 R. AGache, La Somme romaine el préromaine, 1978, p. $387 \mathrm{et} s q$. 